\begin{tabular}{ccc}
\hline & International Journal of Engineering \& Technology, $7(2.7)(2018) 243-245$ \\
SPC & International Journal of Engineering \& Technology \\
Website: $w w w . s c i e n c e p u b c o . c o m / i n d e x . p h p / I J E T$ & Research Paper \\
\hline
\end{tabular}

\title{
Simplified partial resampling method for state estimation using particle filter
}

\author{
M. Tirumala Reddy ${ }^{1}$, Y. Sri Ganesh ${ }^{1}$, Ch. Lakshmi Gayathri ${ }^{1}$, T. Megha Shyam ${ }^{1}$, \\ S. Koteswar Rao ${ }^{2}$, V. Gopi Tilak ${ }^{3}$ \\ ${ }^{1}$ B.TECH Student, K. L. E. F, Vaddeswaram, Guntur. \\ ${ }^{2}$ Prof. Department of E.C.E, K.L. E. F, Vaddeswaram, Guntur \\ ${ }^{3}$ M.TECH student, K.L. E. F, Vaddeswaram, Guntur \\ *Corresponding author E-mail: tirumalareddy.madina@gmail.com
}

\begin{abstract}
Particle filter methods are used in the estimation and tracking of the objects for non-linear and non-gaussian noise conditions. In this paper work the object estimation using partial resampling methods are discussed. On using partial resampling method resampling becomes faster. The performance of particle filter with partial resampling scheme is analyzed using the state estima-tion of a simple pendulum.
\end{abstract}

Keywords: Particle Filters; Partial Resampling; Simple Pendulum; State Estimation.

\section{Introduction}

In optimal filtering method, the state of time varying objects are estimated optimally. In Bayesian filtering method, the state of the object is estimated based on the dynamic variables like velocity, acceleration, orientation etc., [1].The solution to the Bayesian inference equation is obtained by Monte Carlo methods, this performs computation by taking samples from distribution and the quantities will be estimated by the averaging of samples [2]. Estimation in Monte-Carlo approximation is estimated by independent random variables [4], [5]. During the collection of samples, degeneration problem occurs, where the collected particles may have zero weights or some particles having huge weights [2]. This is avoided, by obtaining $\mathrm{N}$ new samples replacing the previous samples from the discrete distribution defined by the weights. This process is called as Resampling. In this paper the corresponding work is to implement the resampling using the particle filter. By choosing the nonlinear system and considering the nonlinear equations of the pendulum and implemented the particle filter on that system.

The basic idea of particle filters are generation of new particles, calculate weight of particles, and resampling process[6]. The resampling process is hard in every implementation of particle filtering because without it, the weights of the particles are varied, then the assumption is made by using only a very small number of particles. The main idea of resampling is removing particle trajectories having small weights and replace them with trajectories with large weights [6].

\section{Particle filter}

This is a primeval model which works well immensely on nonlinear systems, which is problematic for Kalman filter. This was designed by the work of Sir. Nicolas Metropolis and Sir. Norbert Wiener in 1949 by investigating sets of particles rather than individual species[1].
Even though the EKF (Extended Kalman Filter) works for certain nonlinear data, it cannot process systematically, the nonlinearity is high because it relies on linearization of mean and covariance of propagation. First order accuracy was followed by EKF, UKF follows higher order accuracy, here comes the irreducible divergence, if the nonlinearity is still severe. So, the particle filter is a perfect suit for nonlinear system tracking. Particle filter primarily depends on probabilities for estimation. Bayesian state estimator stands as a basis for the particle filter.

Hence the aim of particle filtering is to resample the particles based on their weights. At first, the discrete distribution of pendulum measurements should be considered. The measurements of the pendulum are calculated based on the distance of the bob from the ground. The most likely and unlikely particles are separated by each particle from a discrete distribution. The categorization of estimating likely and unlikely particles is that the likely particles are those whose measurements are identical to the position of the bob from the ground. The likely particles will be assigned with huge weights compared to unlikely particles. The unlikely particles are not important for sampling. The resampling is done to remove those unlikely particles and to add more number of likely particles so that the movement of the pendulum optimally is estimated.

The main procedure of resampling is to replace the small weighted particles with large weights, this adds extra variance to the estimates. So proper resampling techniques should be followed. This algorithm provides an optimal solution for resampling with less variance.

By the adaptive resampling technique, it select the required sample size based on the variance of particle

$\mathrm{n}_{\text {effective }}=\frac{1}{\sum_{\mathrm{i}=1}^{\mathrm{N}}(\mathrm{B}) 2}$

$\mathrm{B}=\mathrm{M}_{\mathrm{k}}{ }^{(\mathrm{i})}$-normalized weight of $\mathrm{i}^{\prime}$ th particle at $\mathrm{k}$ th time step.

Resampling is made whenever the adequate number of particles is less than that of total number of particles. There sampling procedure is done in three steps: 
1) First identify the weight of each samples $M_{k}$ (i)which is the probability of obtaining the $i$ th sample in the distribution \{ $\left.\mathrm{y}_{\mathrm{k}}(\mathrm{i})\right\}$ where, $\mathrm{i}=1,2,3,4,5,6 \ldots . \mathrm{N}$

2) Remove the samples whose weights are nearly to zero and add new samples that are likely to the measurement using resampling.

3) After resampling set the weights of new samples and old samples as constant say, $M_{k}(i)=1 / N$. Since there is no resampling of the particles at every time step of estimation of pendulum position, it adds additional variance to the estimations.

- At first draw, the prior samples $Z^{(i)}$ given by

$\mathrm{Z}^{(\mathrm{i})}=\mathrm{P}\left(\mathrm{Y}_{0}\right)$

Where $\mathrm{i}=1,2 \ldots \mathrm{N}$ and set the effectiveweight, $\mathrm{MK}_{\mathrm{K}}{ }^{(\mathrm{I})}=1 / \mathrm{N}$ of $\mathrm{i}$ 'th particle at time step $\mathrm{k}$

- $\quad$ For each step of $\mathrm{k}$ draw the new samples from a distribution called importance distribution $\pi(\mathrm{Y} \mid \mathrm{Z} 1: \mathrm{K})$ denoted by

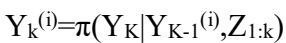

Where $\mathrm{i}=1,2 \ldots \mathrm{N}$ The whole process is called as Sequential importanceresampling (SIR) because here adds a resampling step to each importance sampling. The weight of each particle is estimated by the whole process is called as Sequential importance resampling (SIR) because here resampling step is added to each importance sampling.

The weight of each particle is estimated by

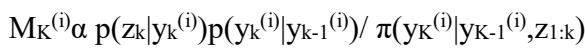

Normalise the weight of each sample to unity. If thenumber of resultant particles are too low, then perform resampling. The following approximation used in filtering distribution is

$\mathrm{P}(\mathrm{yk} \mid \mathrm{Z} 1: \mathrm{k})=\sum_{\mathrm{i}=1}^{\mathrm{N}} \mathrm{M}_{\mathrm{k}}{ }^{(\mathrm{i})} \delta\left(\mathrm{Y}_{\mathrm{k}}-\mathrm{Y}_{\mathrm{k}}{ }^{(\mathrm{i})}\right)$

Performance of SIR depends on the quality of importance distribution $\pi\left(\mathrm{Y} \mid \mathrm{Z}_{1: \mathrm{k}}\right)$.

\section{Pendulum basic eqations}

The differential equation for a simple pendulum of unit mass and unit length is given as [2]

$d^{2} \beta / d t^{2}=-g \sin (\beta)+m(t)$

Where

$\beta$-angle made by the equilibrium axis with respect to pendulum rod's displacement

g- Gravitational acceleration

$\mathrm{m}(\mathrm{t})$-random noise

This mode in terms of state space model can be represented a

$\frac{\mathrm{d}}{\mathrm{dt}}\left(\begin{array}{l}\mathrm{q} 1 \\ \mathrm{q} 2\end{array}\right)=\left(\begin{array}{c}\mathrm{q} 2 \\ -\mathrm{gsinq} 1\end{array}\right)+\left(\begin{array}{l}0 \\ 1\end{array}\right) \mathrm{m}(\mathrm{t})$

Whereq $1=\beta$ andq $2=\mathrm{d} \beta / \mathrm{dt}$

The measurement along the horizontal position of the pendulum leads to a nonlinear measurement model, given by

$\mathrm{Z}_{\mathrm{k}}=\sin \left(\theta\left(\mathrm{t}_{\mathrm{k}}\right)\right)+$ noise

Now the continuous nonlinear state with respect to discrete nonlinear measurements can be discretized as

$\mathrm{Y}_{\mathrm{K}}=\mathrm{F}\left(\mathrm{m}_{\mathrm{k}-1}, \mathrm{q}_{\mathrm{k}-1}\right)$

$\mathrm{Z}_{\mathrm{K}}=\mathrm{H}\left(\mathrm{Y}_{\mathrm{k}}, \mathrm{Z}_{\mathrm{K}}\right)$
Where $\mathrm{Z}_{\mathrm{k}}$ is the measurement vector.

The pendulum model is simply discretized as follows

$$
\left(\begin{array}{l}
\mathrm{X} 1, \mathrm{k} \\
\mathrm{x} 2, \mathrm{k}
\end{array}\right)=\left(\begin{array}{c}
\mathrm{x} 1(\mathrm{k}-1)+\mathrm{x} 2(\mathrm{k}-1) * \Delta \mathrm{T} \\
\mathrm{x} 2(\mathrm{k}-1)-\mathrm{g} * \sin (\mathrm{x} 1(\mathrm{k}-1) * \Delta \mathrm{T}
\end{array}\right)+\mathrm{q}_{\mathrm{k}-1}
$$

$\mathrm{Z}_{\mathrm{k}}=\sin (\mathrm{x} 1(\mathrm{k}))+\mathrm{r}_{\mathrm{k}}$

Where qk-1 $\sim \mathrm{N}$ and $\mathrm{rk} \sim \mathrm{N}(0, \mathrm{R})$ are noise vectors to be considered. Jacobian matrices of $\mathrm{f}$ and $\mathrm{h}$ for first order system is as follows

$\mathrm{Q}=\mathrm{q}^{\mathrm{c}}\left(\frac{\mathrm{dT} * \mathrm{dT} * \mathrm{dT}}{3} \frac{\mathrm{dT} * \mathrm{dT}}{2} ; \frac{\mathrm{dT} * \mathrm{dT}}{2} \mathrm{dT}\right)$

Where qc is the spectral density of continuous time process noise. From equation (3) the weights for pendulum state can be computed

$\mathrm{M}=-1 / \mathrm{e}^{2 \mathrm{R}(\mathrm{y}(\mathrm{k})-\sin (\mathrm{x}(\mathrm{k}, \mathrm{i})) 2}$

Where

K-State; $\mathrm{i}-1,2 \ldots \mathrm{N} ; \mathrm{R}=$ variance

Normalize the $\mathrm{M}$ by

$\mathrm{M}_{\mathrm{i}}=\mathrm{M}_{\mathrm{i}} /\left(\sum_{\mathrm{j}=0}^{\mathrm{n}} \mathrm{M}_{\mathrm{j}}\right)$

\section{Partial resampling}

The methodology of partial resampling is to perform resampling only on particles having high weights and replace them with particles with not cosiderable weights. Particles having moderate weights are not resampled [6]. The advantages of the partial resampling are

1) Partial resampling is performed on smaller number of particles, so resampling becomes faster.

2) In this method less particles are replicated and replaced communication is shorter.

In partial resampling method, resampling is introduced by a step that combines the particles according to their weights in three sets. The weight of each particle is compared with a high and a low thresholds, $\mathrm{R}_{\mathrm{h}}$, and $\mathrm{R}_{\mathrm{l}}$, respectively. Particles having weights between those two thresholds are considered as moderate weights and these weights are not resampled. Now the number of particles with weights greater than $R_{h}$ and less than $R_{L}$ be denoted by $N_{h}$ and $N_{l}$, respectively. The sum of weights of the particles that are resampled is counted using $A_{h l}=\sum_{j=1}^{N h+N l} M(j)$, here $j$ is chosen on the conditions

$\mathrm{M}^{(\mathrm{j})}<\mathrm{R}_{\mathrm{L}}$ or $\mathrm{M}^{(\mathrm{j})}>\mathrm{R}_{\mathrm{h}}$

It consists of two loops. The first loop contains $\mathrm{N}$ iterations and is used for classifying the particles as leading, moderate, or not considerable. The second loop has $\left(\mathrm{N}_{\mathrm{l}}+\mathrm{N}_{\mathrm{h}}\right)$ iterations, which is equal to the number of particles involved in the resampiing. Now a new random measure is produced, and it is given byM ${ }_{t}^{(j)}=1 / A_{h l}$, for ${ }_{t}(j)$ $<\mathrm{R}_{\mathrm{L}}$ OR $\mathrm{M}_{\mathrm{t}}{ }^{(\mathrm{j})}>\mathrm{R}_{\mathrm{H}}$ and $\mathrm{M}_{\mathrm{t}}{ }^{(\mathrm{j})}=\mathrm{M}_{\mathrm{t}-1}(\mathrm{j})$ in other conditions. 


\section{Results}

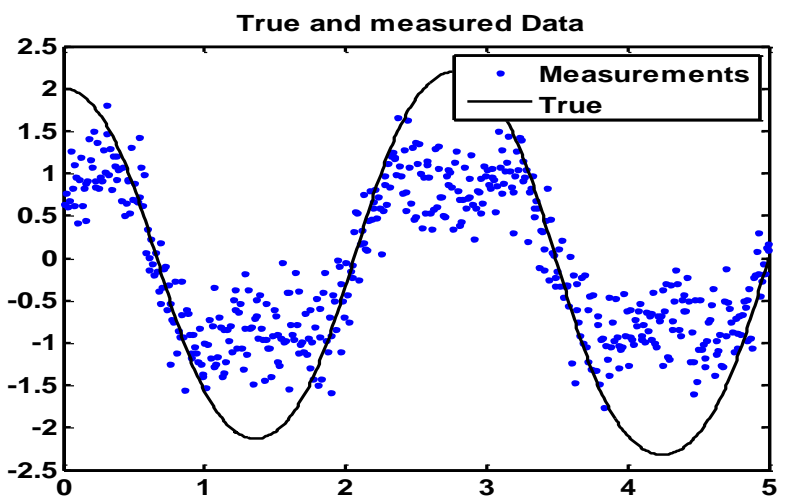

Fig. 1: The Black Line Indicates the True Position of Pendulum. The Blue Dots Represent the Measured Values. The Blue Dotted Line Indicates the Estimated Result Obtained Using Resampling.

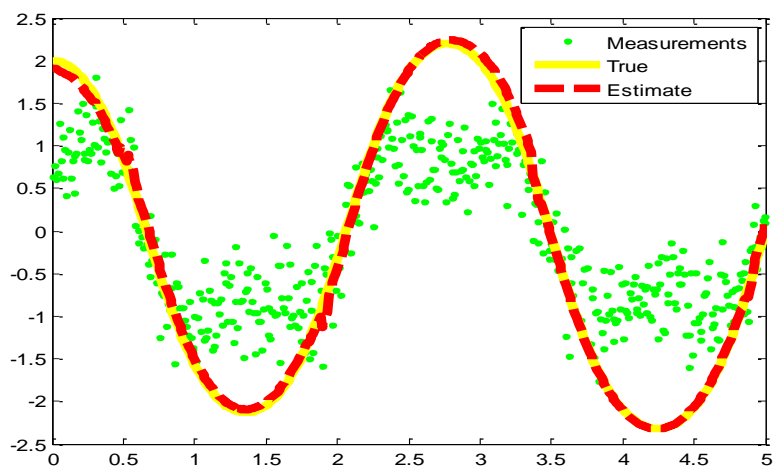

Fig. 2: The Yellow Line Indicates the True Position Value of the Pendulum and the Green Dots Represents the Measured Value of Pendulum at Particular Position. The X Axis Is Taken as Time Axis and Y Axis As A Pendulum Position.

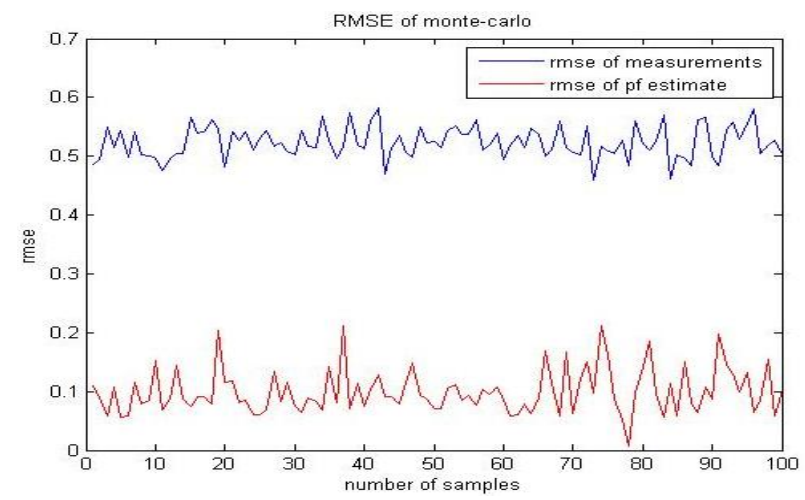

Fig. 3: RMSE of 100 Monte-Carlo results.

\section{Conclusion}

The pendulum state space model has nonlinearities, so by using particle filter for optimal estimation of the position of the pendulum the conclusion as follows, the root mean square error of measurement value is 0.5022 and the particle filter root mean square error is 0.0846 . This implies, Particle filter gives the optimal estimation of the system.

\section{References}

[1] Dan Simon, "Optimal State Estimation Kalman, Ho, and Nonlinear Approaches", John Wiley and sonsinc, publishers.

[2] Simo Sarkka, "Bayesian Filtering and Smoothing", Cambridge University Press.

[3] Simo Sarkka, "Bayesian Estimation of Time-Varying Systems", Copyright (C) Simo Sarakka, 2009-2012.
[4] G.Kitagawa, "Monte Car10 filter and smoother for nonGaussiannonlinear state space models," Journal ofComputational and Graphical Statistics, 5(1) pp. 1-25 (1996)

[5] Doucet, A., De Freitas, N., and Gordon, N. 2001. Sequential Monte Carlo Methods in Practice. Springer.

[6] Sangjin Hong. "New resampling algorithms for particle filters", 2003 IEEE International Conference on Acoustics Speech and Signal Processing 2003 Proceedings (ICASSP 03) ICASSP-03, 2003. 